\title{
EXPONENTIAL CONVERGENCE RATES FOR THE LAW OF LARGE NUMBERS
}

\author{
BY \\ LEONARD E. BAUM(1), MELVIN KATZ( (2), AND ROBERT R. READ(2)
}

Introduction. Consider a sequence of random variables $\left\{X_{k}: k=1,2, \cdots\right\}$ obeying the law of large numbers, i.e., there exists a constant $c$ such that for every $\epsilon>0$ the sequence of probabilities $P\left\{\left|n^{-1} \sum_{k=1}^{n} X_{k}-c\right|>\epsilon\right\}=P_{n}(\epsilon)$ converges to zero as $n \rightarrow \infty$. The object of the present paper is to study the relationships among an exponential convergence rate (i.e., $P_{n}(\epsilon)=O\left(\rho^{n}\right)$ for $\rho=\rho(\epsilon)<1)$, the existence of the individual moment generating functions and the stochastic structure of the sequence $\left\{X_{k}\right\}$. Papers containing related studies (e.g., $[1 ; 3 ; 5])$ have treated the case of independent random variables and demonstrated exponential convergence under the hypothesis that the moment generating functions exist.

The present paper studies the extent to which an exponential convergence rate implies the existence of the moment generating function and conversely. In particular, satisfactory necessary and sufficient conditions (Theorem 2) are found for exponential convergence of sequences of independent (not necessarily identically distributed) random variables.

In the first section it is proved that an exponential rate of convergence for any stationary sequence necessarily implies the existence of the moment generating function of the random variables. Conversely an example is constructed showing that restrictions on the size of the variables cannot be suffcient to insure exponential convergence for the general stationary sequence. The terms size, smallness, etc., as used throughout the paper, refer essentially to the tail probabilities. The case of independence is treated in $\$ 2$ where it is shown that the existence of all the moment generating functions on the same interval is necessary and that a growth restriction on the product of the first $n$ of these generating functions is necessary and sufficient. In $\S 3$ it is shown that the existence of the moment generating functions is necessary for exponential convergence of the averages of a function of the variables of a Markov sequence having stationary transition probabilities. If the process satisfies Doeblin's condition then this condition is proved sufficient. $\$ 4$ contains an example showing that the existence of moment generating functions

Received by the editors May 22, 1961.

(1) Research carried out in the Department of Mathematics, University of Chicago, with partial support of N.S.F. Grant G 14464.

(2) Research carried out in the Department of Statistics, University of Chicago, with the partial support of the Office of Naval Research, Contract Nonr-2121(09). 
generally is not necessary for broader classes of processes. The particular variables constructed are martingale differences, square summable (thus orthogonal) and form a Markov sequence. In the final section exponential convergence to $+\infty$ is treated. An example shows that under the natural definition of such convergence we cannot extend the result of the first section to assert the existence of the moment generating function of $X_{1}^{-}$when $\left\{X_{k}\right\}$ is an ergodic stationary process.

1. Stationary random variables. Let $\left\{X_{k}: k=1,2, \cdots\right\}$ be an arbitrary sequence of random variables.

Definition. The sequence $\left\{X_{k}: k=1,2, \cdots\right\}$ converges exponentially rapidly to the constant $c$ if for all $\epsilon>0$ there exist constants $A$ and $\rho<1$ (depending on $\epsilon$ ) such that

$$
P\left\{\left|n^{-1} \sum_{k=1}^{n} X_{k}-c\right| \geqq \epsilon\right\} \leqq A \rho^{n}, \quad n=1,2, \cdots .
$$

THEOREM 1. Let $\left\{X_{k}: k=1,2, \cdots\right\}$ be a stationary sequence of random variables such that (1) is valid for some $c, \epsilon>0, A$ and $\rho<1$. Then there exists $t_{0}>0$ such that

$$
E e^{t X_{1}}<\infty \quad \text { for } t \in\left[-t_{0}, t_{0}\right] .
$$

Proof. We assume, with no loss of generality, that $c=0$. First observe that

$$
\begin{aligned}
& P\left\{X_{1} \geqq(2 n-1) \epsilon \text { and } \sum_{k=2}^{n} X_{k} \geqq-(n-1) \epsilon\right\} \\
& \geqq P\left\{X_{1} \geqq(2 n-1) \epsilon\right\}+P\left\{\sum_{k=2}^{n} X_{k} \geqq-(n-1) \epsilon\right\}-1 .
\end{aligned}
$$

It follows from stationarity that

$$
P\left\{\sum_{k=2}^{n} X_{k} \geqq-(n-1) \epsilon\right\} \geqq 1-A \rho^{n-1}
$$

and thus (2) yields

$$
\begin{aligned}
P\left\{X_{1} \geqq(2 n-1) \epsilon \text { and } \sum_{k=2}^{n} X_{k} \geqq-(n-1) \epsilon\right\} & \\
& \geqq P\left\{X_{1} \geqq(2 n-1) \epsilon\right\}-A \rho^{n-1} .
\end{aligned}
$$

By hypothesis $P\left\{X_{1} \geqq(2 n-1) \epsilon \quad\right.$ and $\left.\quad \sum_{k=2}^{n} X_{k} \geqq-(n-1) \epsilon\right\}$ $\leqq P\left\{\sum_{k=1}^{n} X_{k} \geqq n \epsilon\right\} \leqq A \rho^{n}$ and thus from (4) we obtain

$$
P\left\{X_{1} \geqq(2 n-1) \epsilon\right\} \leqq A \rho^{n}+A \rho^{n-1} \leqq 2 A \rho^{n-1} .
$$

This bound on the measure of the sets where $X_{1}^{+}$is large implies the existence of a moment generating function for $X_{1}^{+}$in some nondegenerate interval 
about 0 . The above proof may now be repeated with the events $\left\{X_{1} \leqq-(2 n-1) \epsilon\right\}$ and $\left\{\sum_{k=2}^{n} X_{k} \leqq(n-1) \epsilon\right\}$ replacing $\left\{X_{1} \geqq(2 n-1) \epsilon\right\}$ and $\left\{\sum_{k=2}^{n} X_{k} \geqq-(n-1) \epsilon\right\}$ respectively, thus yielding a proof for the existence of the moment generating function of $X_{1}^{-}$in a nondegenerate interval about 0 . This completes the proof since $E e^{t X_{1}} \leqq E e^{t X_{1}^{+}}+E e^{-t X_{1}^{-}}$.

The proof of the above theorem can be applied using rates of convergence other than exponential. The rate controls the size of the $X_{k}$. The almost everywhere convergence of $n^{-1} \sum_{k=1}^{n} X_{k}$ alone does not insure the smallness of the $X_{k}$ (i.e., the converse of the ergodic theorem is false [6, p. 32]) essentially because of the possibility of cancellation of positive and negative values of the $X_{k}$. Other conditions under which convergence will insure the smallness of the $X_{k}$ include (i) assuming the $X_{k}$ to be semi-integrable [ibid.], and (ii) making assumptions on the joint distributions, e.g., independence (converse of the strong law of large numbers).

The following example demonstrates that an exponential rate of convergence for a stationary stochastic process $\left\{X_{k}\right\}$ does not follow from the smallness of the $X_{k}$ and any properties (e.g., mixing properties) of the associated shift transformation generating the process but is essentially a property of the joint distributions themselves.

Let $T$ be an ergodic measure preserving transformation on $(\Omega, Q, P)$ and $\left\{A_{i}: i=1,2, \cdots\right\}$ be a sequence of measurable subsets of $\Omega$ such that $P\left(A_{i}\right)=1 / i^{3}$. Define $A=\bigcup_{i=2}^{\infty} \bigcup_{j=0}^{i-1} T^{j} A_{i}$. Then $P(A) \leqq \sum_{i=2}^{\infty} i / i^{3}=\pi^{2} / 6-1$ and if $\chi_{A}$ denotes the characteristic function of the set $A$, we have $E\left(\chi_{A}\right)<1$. However, for $n=2,3, \cdots$

$$
P\left\{n^{-1} \sum_{j=0}^{n-1} \chi_{A}\left(T^{j}\right)=1\right\} \geqq P\left(A_{n}\right)=n^{-3} .
$$

Thus since $n^{-3}$ is eventually greater than $A \rho^{n}$ for any constants $A$ and $\rho<1$, an exponential rate of convergence for the stationary $(0,1)$ process $\left\{\chi_{A}\left(T^{j}\right)\right.$ $\left.\equiv X_{j}: j=0,1, \cdots\right\}$ to its limit $\left(\pi^{2} / 6-1\right)$ is impossible for any $\epsilon<1$ $-\left(\pi^{2} / 6-1\right)$.

2. Independent random variables. Analogous to Theorem 1 we have the following lemma.

Lemma. Let $\left\{X_{k}: k=1,2, \cdots\right\}$ be a sequence of independent random variables such that (1) holds for some constants $c, A, \epsilon>0$ and $\rho<1$. Then each $X_{k}$ has a moment generating function and these moment generating functions all exist on some common interval about zero.

Proof. Choosing $c=0$ as previously, we note that

$$
A \rho^{n} \geqq P\left\{\sum_{k=1}^{n} X_{k} \geqq 2 n \epsilon\right\} \geqq P\left\{X_{j} \geqq 4 n \epsilon\right\}-P\left\{\sum_{k=1 ; k \neq j}^{n} X_{k} \leqq-2 n \epsilon\right\}
$$


for all $n \geqq j$. From the assumption of independence it follows that

$$
P\left\{\sum_{k=1 ; k \neq j}^{n} X_{k} \leqq-2 n \epsilon\right\} P\left\{X_{j} \leqq n \epsilon\right\} \leqq P\left\{\sum_{k=1}^{n} X_{k} \leqq-n \epsilon\right\} \leqq A \rho^{n} .
$$

Let $1>\delta>0$. For each $j$ there exists an $n_{j}$ sufficiently large so that $P\left\{X_{j} \leqq n \epsilon\right\} \geqq \delta$ for all $n \geqq n_{j}$. Thus from (6) and (7) we obtain

$$
P\left\{X_{j} \geqq 4 n \epsilon\right\} \leqq \frac{A}{\delta} \rho^{n}+A \rho^{n} \leqq \frac{2 A}{\delta} \rho^{n}=B \rho^{n}
$$

for $n \geqq n_{j}$. Since for $t>0, E e^{t X_{j}^{+}} \leqq 1+\sum_{k} e^{(4 k+1) t \epsilon} P\left\{X_{j} \geqq 4(k-1) \epsilon\right\}$ $\leqq 1+\sum_{k=1}^{n_{j}+1} e^{(4 k+1) t e}+B e^{t_{e}} \rho^{-1} \sum_{k=1}^{\infty}\left[e^{4 t e} \rho\right]^{k}<\infty$ if $e^{4 t e} \rho<1$ and a similar argument provides the same estimate for $E e^{t \boldsymbol{X}_{j}^{-}}$it follows that $E e^{t \boldsymbol{X}_{j}}<\infty$ for all $t \in\left[-t_{0}, t_{0}\right]$ with $t_{0}$ determined independently of $j$.

Observe then that an exponential rate of convergence of an independent sequence $\left\{X_{k}\right\}$ (or even (1) for a single $\epsilon$ and $\rho<1$ ) implies the smallness of each $X_{k}$. This result contrasts with the situation in, say, almost everywhere convergence in which any finite number of the $X_{k}$ may be changed arbitrarily without affecting convergence.

TheOREM 2. Let $\left\{X_{k}: k=1,2, \cdots\right\}$ be a sequence of independent random variables and let $S_{n}=\sum_{1}^{n} X_{k}$. Then the sequence $\left\{X_{k}\right\}$ converges exponentially fast to some constant $c$ if and only if for all $\epsilon>0$ there exists a constant $M_{c}$ and $t_{\epsilon}>0$ such that $\prod_{k=1}^{n} E e^{t\left(x_{k}-c\right)}=E e^{t\left(S_{n}-n c\right)} \leqq M_{\epsilon} e^{|t| \epsilon n}$ for $t \in\left[-t_{\epsilon}, t_{\epsilon}\right]$.

Proof. We choose $c=0$ without loss of generality and proceed to the proof of the necessity part of the argument. Given $\epsilon>0$ take $0<\delta<\epsilon$ and write

$$
E e^{t S_{n}^{+}} \leqq 1+\sum_{k=0}^{\infty} e^{t(k+1) \delta} P\left\{S_{n} \geqq k \delta\right\}, \quad t>0 .
$$

Partitioning the right-hand side of (9) into blocks of length $n$ and employing the inequality

$$
\sum_{k=j n}^{(j+1) n-1} e^{\ell(k+1) \delta} P\left\{S_{n} \geqq k \delta\right\} \leqq n e^{t(j+1) n \delta} P\left\{S_{n} \geqq j n \delta\right\}
$$

we obtain

$$
E e^{t S_{n}^{+}} \leqq 1+n\left[e^{i n \delta}+\sum_{j=0}^{\infty} e^{(j+2) \ell n \delta} P\left\{S_{n} \geqq(j+1) n \delta\right\}\right] .
$$

To estimate $P\left\{S_{n} \geqq(j+1) n \delta\right\}$ we first note that

$$
\begin{aligned}
P\left\{S_{n} \geqq(j+1) n \delta\right\} & \leqq P\left\{S_{n} \geqq(j+1 / 2) n \delta\right\} \\
& \leqq P\left\{S_{j n} \geqq j n \delta / 2\right\}+P\left\{S_{j n}-S_{n} \leqq-(j+1) n \delta / 2\right\}
\end{aligned}
$$


By assumption $\left\{X_{k}\right\}$ converges exponentially fast and hence there exist constants $A_{\delta / 2}$ and $\rho_{\delta / 2}<1$ such that for all $j$

$$
P\left\{S_{j n} \geqq j n \delta / 2\right\} \leqq A_{\delta / 2 \rho_{\delta / 2}}^{j n} \text {. }
$$

To bound the second term on the right-hand side of (11) we note that from the assumption of independence

$$
P\left\{S_{n}<n \delta / 2\right\} P\left\{S_{j n}-S_{n} \leqq-(j+1) n \delta / 2\right\} \leqq P\left\{S_{j n} \leqq-j n \delta / 2\right\}
$$

and thus since $P\left\{S_{n} \leqq n \delta / 2\right\} \geqq 1-A_{\delta / 2} \rho_{\delta / 2}^{n}>\eta>0$ for $n$ sufficiently large, it follows that

$$
P\left\{S_{j n}-S_{n} \leqq-(j+1) n \delta / 2\right\} \leqq A_{\delta / 2 \rho_{\delta / 2} \eta^{n j}}{ }^{-1}
$$

for $n$ large, and $j=1,2, \cdots$. (Note, the necessary largeness of $n$ does not depend on $j$.) Combining (11), (12) and (14) we obtain

$$
P\left\{\dot{S}_{n} \geqq(j+1) n \delta\right\} \leqq B_{\delta} \rho_{\delta / 2}^{n j}
$$

for large $n$ and all $j$ and consequently it follows from (10) that

$$
\begin{aligned}
E e^{t S_{n}^{+}} & \leqq 1+n\left\{e^{t n \delta}+B_{\delta} e^{2 t \delta n} \sum_{j=0}^{\infty} e^{t j n \delta} \rho_{\delta / 2}^{j n}\right\} \\
& \leqq n\left\{2^{t n \delta}+B_{\delta} e^{2 t \delta n} /\left[1-\left(\rho_{\delta / 2} e^{t \delta}\right)^{n}\right]\right\}
\end{aligned}
$$

for large $n$ and positive $t$ such that $\rho_{8 / 2} e^{t \delta}<1$. Consequently it is clear that there exists a constant, say $d_{\delta}$, such that

$$
E e^{t S_{n}^{+}} \leqq n d_{\delta} e^{2|t| n \delta}
$$

for $t$ in some interval about zero. It is clear that an analogous argument shows

$$
E e^{t S_{n}^{-}} \leqq n d_{\delta}^{\prime} e^{2|t| n \delta}
$$

for $t$ in some interval about zero. Thus it follows that there exist $M_{\text {e }}$ and $t_{\epsilon}>0$ such that $E e^{t S_{n}} \leqq M_{\epsilon} e^{|t| \epsilon n}$ for $t \in\left[-t_{\epsilon}, t_{\epsilon}\right]$ and necessity is proved.

For the sufficiency we use the well-known inequality $[9$, p. 158]

$$
P\left\{S_{n} \geqq n \epsilon\right\} \leqq E e^{t\left(S_{n}-n \epsilon\right)}
$$

for $t>0$.

Choosing $\delta<\epsilon$ we obtain $P\left\{S_{n} \geqq n \epsilon\right\} \leqq \exp \left(-t_{\delta} n \epsilon\right) M_{\delta} \exp \left(t_{\delta} n \delta\right)=M_{\delta}[\exp$ $\left.t_{\delta}(\delta-\epsilon)\right]^{n}$; a similar argument shows that $P\left\{S_{n} \leqq-n \epsilon\right\}$ converges to zero exponentially fast and this completes the proof.

The above necessary and sufficient conditions are "satisfactory" (compare $[9$, p. 258]) in that they involve functions of quantities associated with the individual random variables themselves, namely products of integrals of 
their exponentials, rather than functions of quantities associated with the sums.

If the random variables $\left\{X_{k}\right\}$ are Gaussian, the previous condition can be simply expressed in terms of the means and variances.

Corollary. A sequence of independent Gaussian random variables converges exponentially rapidly to $c$ if and only if the sequence of averages of expected values converge to $c$ and the sequence of averages of the variances is bounded.

3. Markov processes. In this section we shall consider a Markov process $\left\{X_{k}: k=0,1,2, \cdots\right\}$ with state space $(\mathfrak{X}, \mathfrak{B})$ and stationary transition probability function $p(x, B)$. Let $f$ denote a real valued measurable function on the state space. In what follows we shall be interested only in exponential rates of convergence that are independent of an arbitrary initial probability measure $\nu$ on the state space of the process, i.e., the constants $A$ and $\rho<1$ do not depend on $\nu$.

TheOREM 3. Let $\left\{X_{k}: k=0,1,2, \cdots\right\}$ be a Markov process as described above. If $P\left\{\left|n^{-1} \sum_{k=1}^{n} f\left(X_{k}\right)-c\right| \geqq \epsilon \mid x_{0}\right\} \leqq A \rho^{n}$ for all $x_{0}$, for some $c, \epsilon>0, A$ and $\rho<1$, then for each $k \geqq 1$ the moment generating functions $E_{x_{0}} e^{t f\left(x_{k}\right)}$ exist and are finite for $t \in\left(-t_{0}, t_{0}\right)$, with $t_{0}>0$ not depending on $x_{0}$ or $k$, and where $E_{x_{0}} e^{t f\left(x_{k}\right)}$ denotes the expected value with initial measure assigning probability one to $x_{0}$.

Proof. The pattern of proof of Theorem 1 will be followed. The probabilities in the inequalities (2) through (5) should now be interpreted as conditional probabilities given $X_{0}=x_{0}$. Again take $c=0$. Now (3) follows from

$$
\begin{aligned}
P\left\{\sum_{k=2}^{n} f\left(X_{k}\right) \leqq-(n-1) \epsilon \mid\right. & \left.x_{0}\right\} \\
& =E\left\{P\left\{\sum_{k=2}^{n} f\left(X_{k}\right) \leqq-(n-1) \epsilon \mid X_{1}\right\} \mid x_{0}\right\} \\
& \leqq E\left\{A \rho^{n-1} \mid x_{0}\right\} \\
& =A \rho^{n-1} \quad \text { for all } x_{0} .
\end{aligned}
$$

Thus as in the proof of Theorem 1 it follows that $E\left\{e^{t f^{+}\left(x_{1}\right)} \mid x_{0}\right\}<\infty$ for $t$ in some nondegenerate interval (independent of $x_{0}$ ) about zero. Consequently the moment generating function of $f^{+}\left(X_{1}\right)$ for arbitrary initial measure exists in this interval. Also the above argument shows that the conditional moment generating functions of the $f^{+}\left(X_{j}\right)$ given $X_{j-1}$ are uniformly bounded in the $X_{j-1}$, and it follows that for $j>1$

$$
E\left\{e^{t f^{+}\left(X_{j}\right)} \mid x_{0}\right\}=E\left\{E\left\{e^{t f^{+}\left(X_{j}\right)} \mid X_{j-1}\right\} \mid x_{0}\right\}
$$

and hence the moment generating function of $f^{+}\left(X_{j}\right)$ exists on the same 
$t$-interval as the moment generating function of $f^{+}\left(X_{1}\right)$. Clearly we can show the existence of the moment generating function for $f^{-}\left(X_{j}\right)$ in an analogous manner and this completes the proof.

It is known that in general the converse of Theorem 3 is not valid and that in particular, it cannot be assured for stationary Markov processes. It can be demonstrated however for processes whose transition probabilities satisfy Doeblin's condition [4]. For convenience assume that the process $\left\{X_{k} ; k=0,1,2, \cdots\right\}$ has only one ergodic class. Denote the expected value of $f\left(X_{0}\right)$ with respect to the (now) unique stationary measure on $\mathfrak{X}$ by $\mu$.

Theorem 4. Let $\left\{X_{k}: k=0,1,2, \cdots\right\}$ denote the Markov process determined by $p(x, B)$, satisfying Doeblin's condition. Then for every $\epsilon>0$ $P\left\{\left|n^{-1} \sum_{k=1}^{n} f\left(X_{k}\right)-\mu\right| \geqq \epsilon \mid x_{0}\right\} \leqq A \rho^{n}$ for $n=1,2, \ldots$ and $A, \rho<1$ independent of $x_{0}$, if and only if there exists $A_{1}, \rho_{1}<1$ such that $P\left\{\left|f\left(X_{k}\right)\right| \geqq n \epsilon \mid x_{0}\right\}$ $\leqq A_{1} \rho_{1}^{n}$ for $k, n=1,2, \cdots$, independent of $x_{0}$.

Proof. Clearly the proof of Theorem 3 can be modified slightly to give the proof of the necessity of the condition. To prove sufficiency we proceed as in [8]. There is no loss of generality in assuming $\mu<0$. It is enough to show that there exist two constants $C$ and $\gamma<1$, each independent of $x_{0}$ such that for all $n, P\left\{\sum_{k=1}^{n} f\left(X_{k}\right) \geqq 0 \mid x_{0}\right\} \leqq C \gamma^{n},(\epsilon=-\mu)$. Define

$$
\phi(n, t)=\sup _{x_{0} \in \mathfrak{X}} E\left\{\exp \left(t \sum_{k=1}^{n} f\left(X_{k}\right)\right) \mid x_{0}\right\} .
$$

By hypothesis there exists a nondegenerate interval about zero such that for $t$ included in this interval $\phi(n, t)$ is well defined for all $n$. If $n=j+l$ then

$$
\begin{aligned}
E\{\exp [t & \left.\left.\sum_{k=1}^{n} f\left(X_{k}\right)\right] \mid x_{0}\right\} \\
= & E\left\{E\left[\exp \left(t \sum_{k=1}^{j} f\left(X_{k}\right)+t \sum_{k=j+1}^{n} f\left(X_{k}\right)\right) \mid X_{1}, \cdots, X_{j}\right] \mid x_{0}\right\} \\
& =E\left\{\exp \left[t \sum_{k=1}^{j} f\left(X_{k}\right)\right] E\left(\exp \left(t \sum_{k=j+1}^{n} f\left(X_{k}\right)\right) \mid X_{j}\right) \mid x_{0}\right\} \\
& \leqq \phi(j, t) \phi(l, t) .
\end{aligned}
$$

Thus $\phi(n, t) \leqq \phi(j, t) \phi(l, t)$. For any integer $d$ and for $n \geqq d$ write $n=m d+l$, $0 \leqq l \leqq d-1$, and then

$$
\phi(n, t) \leqq \phi(m d, t) \phi(l, t) \leqq[\phi(d, t)]^{m} \phi(l, t) .
$$

Therefore $E\left(\exp \left[t \sum_{k=1}^{n} f\left(X_{k}\right)\right] \mid x_{0}\right)^{1 / n} \leqq[\phi(d, t)]^{m / n}[\phi(l, t)]^{1 / n}$ and it follows, letting $n \rightarrow \infty$, that 


$$
\limsup _{n} E\left(\exp \left[t \sum_{k=1}^{n} f\left(X_{k}\right)\right] \mid x_{0}\right)^{1 / n} \leqq[\phi(d, t)]^{1 / d}
$$

The result will follow if there exist $t_{0}>0$ and an integer $d_{0}$ such that $\phi\left(d_{0}, t_{0}\right)$ $<1$, since $P\left\{\sum_{k=1}^{n} f\left(X_{k}\right) \geqq 0 \mid x_{0}\right\} \leqq E\left\{\exp \left[t_{0} \sum_{k=1}^{n} f\left(X_{k}\right)\right] \mid x_{0}\right\} \leqq C \gamma^{n}$ where $\gamma=\left\{\phi\left(d_{0}, t_{0}\right)^{1 / d_{0}}+\eta\right\}$ and $\eta$ is chosen so that $\gamma<1$.

For sufficiently small $t>0$ consider the limited expansion

$$
\begin{aligned}
& E\left\{\exp \left[t \sum_{k=1}^{d} f\left(X_{k}\right)\right] \mid x_{0}\right\} \leqq 1+t E\left\{d\left(d^{-1} \sum_{k=1}^{d} f\left(X_{k}\right)\right) \mid x_{0}\right\} \\
&+t^{2} E\left\{\left[\sum_{k=1}^{d} f\left(X_{k}\right)\right]^{2} \exp \left[t \sum_{k=1}^{d}\left|f\left(X_{k}\right)\right|\right] \mid x_{0}\right\} .
\end{aligned}
$$

It will be shown that the coefficient of $d t$ in the second term of the righthand side of (25) converges uniformly to $\mu$ and the function multiplying $t^{2}$ in the third term can be dominated independently of $x_{0}$ for sufficiently small positive $t$. Thus $d_{0}$ and $t_{0}$ can be chosen so that $\phi\left(d_{0}, t_{0}\right)<1$. that

First note that three functions $g_{0}(t), g_{1}(t)$ and $g_{2}(t)$ can be found such

$$
\begin{aligned}
E\left\{\exp \left[t\left|f\left(X_{1}\right)\right|\right] \mid x_{0}\right\} & \leqq g_{0}(t)=K_{0}\left(1-\rho_{1} e^{t_{\epsilon}}\right)^{-1} \\
E\left\{f\left(X_{1}\right) \exp \left[t\left|f\left(X_{1}\right)\right|\right] \mid x_{0}\right\} & \leqq g_{1}(t)=K_{1}\left(1-\rho_{1} e^{t \epsilon}\right)^{-2} \\
E\left\{f^{2}\left(X_{1}\right) \exp \left[t\left|f\left(X_{1}\right)\right|\right] \mid x_{0}\right\} & \leqq g_{2}(t)=K_{2}\left(1-\rho_{1} e^{t \epsilon}\right)^{-3}
\end{aligned}
$$

for all sufficiently small positive $t$ and suitably chosen constants $K_{0}, K_{1}$ and $K_{2}$. The existence of the $g$ 's follows from the hypothesis of the theorem upon expanding the exponentials on the right-hand side in power series about zero and summing. These functions are to be used in an iterative evaluation of

$$
\begin{gathered}
E\left\{\left[\sum_{k=1}^{d} f\left(X_{k}\right)\right]^{2} \exp \left[t \sum_{k=1}^{d}\left|f\left(X_{k}\right)\right|\right] \mid x_{0}\right\} \\
=\int e^{t\left|f\left(x_{1}\right)\right|} p\left(x_{0}, d x_{1}\right) \int e^{t\left|f\left(x_{2}\right)\right|} p\left(x_{1}, d x_{2}\right) \cdots \int e^{t\left|f\left(x_{d}\right)\right|} \\
\cdot\left\{\sum_{k=1}^{d} f\left(x_{k}\right)\right\}^{2} p\left(x_{d-1}, d x_{d}\right) .
\end{gathered}
$$

Introducing the notation (for fixed $d$ )

$$
I_{d}\left(x_{d-1}\right)=\int e^{\ell\left|f\left(x_{d}\right)\right|}\left\{f^{2}\left(x_{d}\right)+2 f\left(x_{d}\right) \sum_{k=1}^{d-1} f\left(x_{k}\right)+\left[\sum_{k=1}^{d-1} f\left(x_{k}\right)\right]^{2}\right\} p\left(x_{d-1}, d x_{d}\right)
$$

and $y_{j}=\sum_{k=1}^{j} f\left(x_{k}\right)$ for $j=1, \cdots, d-1$ we obtain

$$
I_{d}\left(x_{d-1}\right) \leqq g_{2}(t)+2 g_{1}(t) y_{d-1}+g_{0}(t) y_{d-1}^{2} .
$$


Define $I_{d-k}\left(x_{d-k-1}\right)=\int e^{t\left|f\left(x_{d-k}\right)\right|} I_{d-k+1}\left(x_{d-k}\right) p\left(x_{d-k-1}, d x_{d-k}\right)$ for $k=1, \cdots, d-1$, then from (28)

$$
\begin{aligned}
I_{d-1}\left(x_{d-2}\right) \leqq & g_{2}(t) g_{0}(t)+2 g_{1}(t) \int e^{t\left|f\left(x_{d-1}\right)\right|}\left[f\left(x_{d-1}\right)+y_{d-2}\right] p\left(x_{d-2}, d x_{d-1}\right) \\
+ & g_{0}(t) \int e^{t\left|f\left(x_{d-1}\right)\right|}\left[f^{2}\left(x_{d-1}\right)+2 f\left(x_{d-1}\right) y_{d-2}+y_{d-2}^{2}\right\} p\left(x_{d-2}, d x_{d-1}\right) \\
\leqq & g_{0}(t) g_{2}(t)+2 g_{1}^{2}(t)+2 g_{1}(t) g_{0}(t) y_{d-2}+g_{0}(t) g_{2}(t) \\
& +2 g_{0}(t) g_{1}(t) y_{d-2}+g_{0}^{2}(t) y_{d-2}^{2} \\
= & 2 g_{0}(t) g_{2}(t)+2 g_{1}^{2}(t)+4 g_{0}(t) g_{1}(t) y_{d-2}+g_{0}^{2}(t) y_{d-2}^{2} .
\end{aligned}
$$

Proceeding inductively we obtain

$$
\begin{aligned}
I_{2}\left(x_{1}\right) \leqq(d-1) g_{0}^{d-2}(t) g_{2}(t) & +(d-2)(d-1) g_{0}^{d-3}(t) g_{1}^{2}(t)+g_{0}^{d-1}(t) y_{1}^{2} \\
& +2(d-1) g_{0}^{d-2}(t) g_{1}(t) y_{1}
\end{aligned}
$$

and finally

$$
\begin{aligned}
E\left\{\left[\sum_{k=1}^{d} f\left(X_{k}\right)\right]^{2} \exp \left[t \sum_{k=1}^{d}\left|f\left(X_{k}\right)\right|\right] \mid x_{0}\right\} & \leqq \int e^{t\left|f\left(x_{1}\right)\right| I_{2}\left(x_{1}\right) p\left(x_{0}, d x_{1}\right)} \\
& \leqq d g_{0}^{d-1}(t) g_{2}(t)+d(d-1) g_{0}^{d-2}(t) g_{1}^{2}(t) .
\end{aligned}
$$

It remains to prove that $E\left\{n^{-1} \sum_{k=1}^{n} f\left(X_{k}\right) \mid x_{0}\right\} \rightarrow \mu$ uniformly in $x_{0}$. Observe that

$$
\begin{aligned}
E\left\{n^{-1} \sum_{k=1}^{n} f\left(X_{k}\right) \mid x_{0}\right\} & =n^{-1} \sum_{k=1}^{n} E\left\{f\left(X_{k}\right) \mid x_{0}\right\} \\
& =n^{-1} \sum_{k=1}^{n} \int f(y) \int p(x, d y) p^{(k-1)}\left(x_{0}, d x\right) \\
& =n^{-1} \sum_{k=1}^{n} \int\left[\int f(y) p(x, d y)\right] p^{(k-1)}\left(x_{0}, d x\right) \\
& =\int\left[\int f(y) p(x, d y)\right] n^{-1} \sum_{k=1}^{n} p^{(k-1)}\left(x_{0}, d x\right)
\end{aligned}
$$

where all integrals are taken over $\mathfrak{X}$ and $p^{(0)}\left(x_{0}, d x\right)$ assigns probability one to the point $x_{0}$. The interchange of the order of integration follows from an extension of the Fubini theorem which is given as problem $13[9$, p. 140] and whose proof is like that of the Fubini theorem. From Doeblin's condition it follows that $n^{-1} \sum_{k=1}^{n} p^{(k-1)}(x, B) \rightarrow \pi(B)$ uniformly in $x$ and $B$, where $\pi(\cdot)$ denotes the unique stationary initial measure. From (26) we have that $\left|\int f(y) p(x, d y)\right| \leqq g_{0}(0)+g_{2}(0)$ and thus it follows that 


$$
E\left\{n^{-1} \sum_{k=1}^{n} f\left(X_{k}\right) \mid x_{0}\right\} \rightarrow \int\left[\int f(y) p(x, d y)\right] \pi(d x)
$$

uniformly in $x_{0}$. However

$$
\begin{aligned}
\int\left[\int f(y) p(x, d y)\right] \pi(d x) & =\int f(y) \int p(x, d y) \pi(d x) \\
& =\int f(y) \pi(d y)=\mu<0
\end{aligned}
$$

and consequently we may choose an integer $d_{0}$ such that

$$
E\left\{d_{0}^{-1} \sum_{k=1}^{d_{0}} f\left(X_{k}\right) \mid x_{0}\right\} \leqq \delta<0 \quad \text { for all } x_{0} .
$$

4. Existence of moment generating functions not necessary. For nonstationary processes the necessity theorems of $\$ \S 2$ and 3 for independent and Markov processes do not extend to other familiar classes of stochastic sequences. Specifically we construct a martingale difference sequence of square summable (thus orthogonal) functions which is also a Markov process (with nonstationary transition probabilities) which converges exponentially rapidly although none of the moment generating functions exist on any interval.

Let $X_{1}=0,2^{n},-2^{n}$ on sets $E_{0}, E_{n}^{+}, E_{n}^{-}$having measures $13 / 15,2^{-4 n}, 2^{-4 n}$ respectively. Thus $E X_{1}^{2}=2 \sum_{n=1}^{\infty} 2^{2 n} 2^{-4 n}=2 / 3$. Next split each $X_{1}$ atom into two sets, e.g. $E_{n}^{++}, E_{n}^{\infty}\left(E_{n}^{+}=E_{n}^{++} \cup E_{n}^{+-}\right)$of equal measure. Define $X_{2}=0$, $2^{n},-2^{n}, 2^{n},-2^{n}$ on the sets $E_{0}, E_{n}^{++}, E_{n}^{+-}, E_{n}^{-+}, E_{n}^{--}$with measures $13 / 15$ for $E_{0}$ and $2^{-4 n-1}$ for each of the others. Thus $E X_{2}^{2}=E X_{1}^{2}$ and $E\left(X_{2} \mid X_{1}\right)=0$. Let each $X_{2}$ atom where $X_{1}+X_{2}=2^{n+1}$ or $-2^{n+1}$ be halved and let $X_{3}=2^{n+1}$ and $-2^{n+1}$ on each such pair (each member having measure $2^{-4 n-2}$ ); and on the set where $X_{1}+X_{2}=0$ (of measure 14/15) let $X_{3}=0$. Thus $E X_{3}^{2}$ $=4 \sum_{1}^{\infty} 2^{2 n+2} 2^{-4 n-2}=4 / 3$ and $E\left[X_{3} \mid X_{2}, X_{1}\right]=E\left(X_{3} \mid X_{2}\right)=0$. In general define, for $j \geqq 2$,

$$
X_{j}=\left\{\begin{array}{c} 
\pm 2^{j-2} 2^{n} \text { on the halves of the set where } \\
\pm 2^{j-2} 2^{n} \text { on the halves of the set where } \\
\sum_{i=1}^{j-1} X_{i}=2^{j-2} 2^{n} \text { each half having measure } 2^{-4 n-j+1} \\
\sum_{i=1}^{i-1} X_{i}=-2^{j-2} 2^{n} \text { each half having measure } 2^{-4 n-j+1} \\
0 \text { on the set where } \sum_{i=1}^{j-1} X_{i}=0 \text { having measure } 1-\frac{2}{15} 2^{-j+2} .
\end{array}\right.
$$


Thus $E X_{j}^{2}=4 \sum_{n=1}^{\infty} 2^{2(j-2)+2 n} 2^{-4 n-j+1}=2^{j-1} / 3(j=2,3, \cdots)$.

On the subset where $X_{j-1} \neq 0$ the $\sigma$-field induced by $X_{j-1}, X_{j-2}, \cdots, X_{1}$ is the $\sigma$-field induced by $X_{j-1}$ itself. Since $X_{j-1}=0$ implies $X_{j}=0,\left\{X_{k}\right\}$ is Markov and a martingale difference sequence: $E\left(X_{j} \mid X_{j-1}, \cdots, X_{1}\right)$ $=E\left(X_{j} \mid X_{j-1}\right)=0$. Since $P\left\{\sum_{k=1}^{j} X_{k} \neq 0\right\}=(4 / 15) 2^{-j}$ the sequence has an exponential convergence rate although for any $j$ and $t \neq 0$

$$
E e^{i X_{i}} \geqq 4 \sum_{n=1}^{\infty} \exp \left(|t| 2^{j-2+n}\right) 2^{-4 n-j+1}=\infty
$$

since the $n$th term becomes infinite. In the reverse direction the example of $\$ 1$ shows that size limitations alone on the random variables do not insure an exponential rate of convergence. It is known however that uniformly bounded sequences will converge exponentially rapidly if they are martingale differences [2]. The sufficiency results of $\$ 2$ suggest the question of exactly what weakening of the uniform boundedness condition suffices to insure an exponential rate of convergence.

5. Convergence to $+\infty$.

Definition. The sequence $\left\{X_{k}: k=1,2, \cdots\right\}$ converges exponentially rapidly to $+\infty$ if for all $M>0$ there exist constants $A$ and $\rho<1$ (depending on $M$ ) such that

$$
P\left\{n^{-1} \sum_{k=1}^{n} X_{k}<M\right\} \leqq A \rho^{n} \quad n=1,2, \cdots .
$$

Proposition. Let $\left\{X_{k}: k=1,2, \cdots\right\}$ be a sequence of independent and identically distributed random variables such that $E X_{1}^{+}=+\infty$ and the moment generating function of $X_{1}^{-}$exists on some interval about 0 . Then $\left\{X_{k}\right\}$ converges to $+\infty$ exponentially rapidly.

Proof. It follows from the hypothesis that

$$
\begin{aligned}
P\left\{n^{-1} \sum_{k=1}^{n} X_{k}^{-}>E\left(X_{1}^{-}\right)+1\right\} & \leqq \prod_{1}^{n} E e^{t\left(X_{k}^{-}-E\left(X_{1}^{-}\right)+1\right)} \\
& \leqq A_{-} \rho_{-}^{n}
\end{aligned}
$$

if $t>0$ is chosen sufficiently close to 0 . Given any constant $Q$, it follows from $E X_{1}^{+}=+\infty$ that

$$
P\left\{n^{-1} \sum_{k=1}^{n} X_{k}^{+}<Q\right\} \leqq \prod_{1}^{n} E e^{t\left(Q-X_{k}^{+}\right)} \leqq A_{+} \rho_{+}^{n}
$$

for $t>0$ sufficiently close to 0 . Then given $M$, 


$$
\begin{aligned}
P\left\{\sum_{k=1}^{n} X_{k}<n M\right\} \leqq & P\left\{\sum_{1}^{n} X_{k}^{+}<n\left(M+E\left(X_{1}^{-}\right)+1\right)\right\} \\
& +P\left\{\sum_{1}^{n} X_{k}^{-}>n\left(E\left(\overline{X_{1}}\right)+1\right)\right\} \\
& \leqq A_{+} \rho_{+}^{n}+A_{-} \rho_{-}^{n} \leqq \mathrm{~B}^{n} .
\end{aligned}
$$

However, exponential convergence to $+\infty$ is not as well behaved as exponential convergence to a finite constant in that a theorem in the converse direction for stationary processes (the analog of Theorem 1) is not available as is demonstrated by the following counterexample.

Specifically we define an ergodic transformation $T$ and a measurable function $f$ so that the stationary sequence $\left\{f \cdot T^{i-1}: i=1,2, \cdots\right\}$ converges exponentially fast to $+\infty$ although $E f^{-}=+\infty$.

Let $T$ be the transformation on $\{[0,1), ৫, P \equiv$ Leb. measure $\}$ that maps $\left[\left(2^{n}-2\right) / 2^{n},\left(2^{n}-1\right) / 2^{n}\right)$ linearly by translation onto $\left[1 / 2^{n}, 2 / 2^{n}\right)$ for $n=1,2, \cdots$. Then $T$ is a one to one, bi-measurable, measure preserving transformation on $[0,1)$ and is also ergodic. (In fact if $E$ is an invariant set of positive measure, then for any $\epsilon>0$ there exists an interval $(a, b)$ such that $P\{E \cap(a, b)\}>(1-\epsilon) P\{(a, b)\}$, and hence there is a diadic interval $\left(i / 2^{n}, j / 2^{n}\right)$ such that $P\left\{E \cap\left(i / 2^{n}, j / 2^{n}\right)\right\}>(1-2 \epsilon) P\left\{\left(i / 2^{n}, j / 2^{n}\right)\right\}$. Then necessarily for some $k, i \leqq k \leqq j, P\left\{E \cap\left(k / 2^{n},(k+1) / 2^{n}\right)\right\} \geqq(1-2 \epsilon) / 2^{n}$. But the transformation $T$ is such that the sets $T^{i}\left(k / 2^{n},(k+1) / 2^{n}\right), i=0,1, \cdots$, $2^{n}-1$, are disjoint and of equal measure so that the invariance of $E$ implies $P\{E\}>2^{n}(1-2 \epsilon) / 2^{n}=1-2 \epsilon$. Thus $P\{E\}=1$ and $T$ is ergodic).

Next we define a function $f$ on $\{[0,1), \infty, P\}$

$$
f(x)=\left\{\begin{array}{lll}
2^{2 n} \cdot 2^{2^{n}} & x \in\left[\left(2^{n}-2\right) / 2^{n},\left(2^{n}-1\right) / 2^{n}\right), & n=2,3, \cdots \\
-2^{n-1} & x \in\left[1 / 2^{n}, 2 / 2^{n}\right), & n=2,3, \cdots
\end{array}\right.
$$

and observe that the transformation $T$ is such that for all $x \in[0,1)$ one of the images $x, T x, \cdots, T^{\left(2^{n-1}-1\right)} x$ is included in $\left[\left(2^{n-1}-1\right) / 2^{n-1}, 1\right)$. Hence at least one of the numbers $f\left(T^{i} x\right), j=0,1, \cdots, 2^{n-1}-1$, is greater than or equal to $2^{2 n} \cdot 2^{2^{n}}$. Therefore for $2^{n-1} \leqq m<2^{n}$, we have that $\sum_{i=0}^{m-1} f\left(T^{i} x\right)$ $\geqq 2^{2 n} \cdot 2^{2^{n}}-\left(2^{n}-1\right)\left(2^{n} 2^{2^{n}}\right)=2^{n} 2^{2^{n}}$, which is eventually greater than $2^{n} M$ for any given $M$, unless one of the summands $f\left(T^{i} x\right)<-2^{n} \cdot 2^{2^{n}}$, that is unless $T^{i} x \in\left[0,1 / 2^{n} \cdot 2^{2^{n}}\right)$ for some $i, i=0, \cdots, m-1$. But $P\left\{T^{i} x \in\left[0,1 / 2^{n} \cdot 2^{2^{n}}\right)\right.$ for some $\left.i=0,1, \cdots, 2^{n}-1\right\} \leqq 2^{n} / 2^{n} \cdot 2^{2^{n}}$ and consequently $P\left\{\sum_{i=0}^{m-1} f\left(T^{i} x\right)\right.$ $\leqq M m\} \leqq(1 / 2)^{m}$ for all sufficiently large $m$. Thus we have an ergodic stationary sequence $\left\{f \cdot T^{i}: i=0,1,2, \cdots\right\}$ converging exponentially fast to $+\infty$ although $E f^{-}=\sum_{n=0}^{\infty} 2^{n-1} 2^{-n}=\infty$. 


\section{REFERENCES}

1. David Blackwell and J. L. Hodges, The probability in the extreme tail of a convolution, Ann. Math. Statist. 30 (1959), 1113-1120.

2. David Blackwell, On optimal systems, Ann. Math. Statist. 25 (1954), 394-397.

3. H. Cramér, Sur un nouveau theoreme-limite de la theorie des probabilites, Actualites Sci. Ind., No. 736, Hermann, Paris, 1938.

4. J. L. Doob, Stochastic processes, Wiley, New York, 1953.

5. W. Feller, Generalisation of a probability limit theorem of Cramer, Trans. Amer. Math. Soc. 54 (1943), 361-372.

6. P. R. Halmos, Lectures on ergodic theory, The Math. Soc. of Japan, Tokyo, 1956.

7. Measure theory, Van Nostrand, New York, 1950.

8. Melvin Katz and A. J. Thomasian, $A$ bound for the law of large numbers for discrete Markoo processes, Ann. Math. Statist. 32 (1961), 336-337.

9. M. Loeve, Probability theory, Van Nostrand, New York, 1960.

University of Chicago,

Chicago, Illinors 